\title{
Dynamic Social Network Modeling of Diffuse Subcellular Morphologies
}

\author{
Andrew Durden" , Allyson T Loy ${ }^{\mathbb{I}}$, Barbara Reaves ${ }^{\ddagger}$, Mojtaba Fazli" , Abigail Courtney ${ }^{\mathbb{I}}$, Frederick D Quinn ${ }^{\ddagger}$, S Chakra \\ Chennubhotla ${ }^{\S}$, Shannon P Quinn ${ }^{\| * * *}$
}

\begin{abstract}
The use of fluorescence microscopy has catalyzed new insights into biological function, and spurred the development of quantitative models from rich biomedical image datasets. While image processing in some capacity is commonplace for extracting and modeling quantitative knowledge from biological systems at varying scales, general-purpose approaches for more advanced modeling are few. In particular, diffuse organellar morphologies, such as mitochondria or actin microtubules, have few if any established spatiotemporal modeling strategies, all but discarding critically important sources of signal from a biological system. Here, we discuss initial work into building spatiotemporal models of diffuse subcellular morphologies, using mitochondrial protein patterns of cervical epithelial (HeLa) cells. We leverage principles of graph theory and consider the diffuse mitochondrial patterns as a social network: a collection of vertices interconnected by weighted and directed edges, indicating spatial relationships. By studying the changing topology of the social networks over time, we gain a mechanistic understanding of the types of stresses imposed on the mitochondria by external stimuli, and can relate these effects in terms of graph theoretic quantities such as centrality, connectivity, and flow. We demonstrate how the mitochondrial pattern can be faithfully represented parametrically using a learned mixture of Gaussians, which is then perturbed to match the spatiotemporal evolution of the mitochondrial patterns over time. The learned Gaussian components can then be converted to graph Laplacians, formally defining a network, and the changes in the topology of the Laplacians can yield biologically-meaningful interpretations of the evolving morphology. We hope to leverage these preliminary results to implement a bioimaging toolbox, using existing open source packages in the scientific Python ecosystem (SciPy, NumPy, scikit-image, OpenCV), which builds dynamic social network models from time series fluorescence images of diffuse subcellular protein patterns. This will enable a direct quantitative comparison of network structure over time and between cells exposed to different conditions.
\end{abstract}

Index Terms—Biomedical Imaging, Graph Theory, Social Networks

\section{Introduction}

Given the recent rise of fluorescence microscopy, and the subsequent proliferation of biomedical imaging data, live cell imaging

II Department of Computer Science, University of Georgia, Athens, GA 30602 USA

II Department of Microbiology, University of Georgia, Athens, GA 30602 USA \$ Department of Infectious Diseases, University of Georgia, Athens, GA 30602 USA

$\S$ Department of Computational and Systems Biology, University of Pittsburgh, Pittsburgh, PA 15232 USA

* Corresponding author: spq@uga.edu

** Department of Cellular Biology, University of Georgia, Athens, GA 30602 USA

Copyright $(2018$ Andrew Durden et al. This is an open-access article distributed under the terms of the Creative Commons Attribution License, which permits unrestricted use, distribution, and reproduction in any medium, provided the original author and source are credited. has become much more accessible. However, the growth in quantification and modeling of biological and biomedical phenomena has been uneven; "solid" morphologies such as cells and nuclei are much easier to automatically segment, track, and quantify than diffuse patterns induced by mitochondria or actin. There is a need for methodologies and software capable of autonomous tracking, segmentation, and quantification of spatiotemporal changes in these structures.

Understanding the spatiotemporal evolution of subcellular organelles in response to external stimuli and modeling this behavior is critical to understanding the effects of the stimuli on the internal state and configuration of the cell. This can have downstream implications in the development of targeted therapies. Recently, spatial covariance has been used to quantify gene expression correlation in image like matrices representing sequenced RNA [STS17]. Other recent work demonstrates the benefits of measuring covariance between subcellular structures to observe how coherent portions of the cells respond in tandem to external stimuli $\left[\mathrm{VCL}^{+} 17\right]$. While this work used handcrafted pixel-level thresholds and manual labeling of pixels into organelle groupings, it nonetheless represents the spirit of our work: developing quantifiable, data-driven spatiotemporal models of subcellular structures.

Our work focuses on both spatial and temporal covariance to better model and understand the response of subcellular structures to stimuli. To do this, we draw on graph theory and cast the punctate subcellular morphologies as instances of a social network. A recent study of brain activity used networks to create a quantitative measure of correlated activity in functional MRI (fMRI) images which could then easily be clustered [DGD $\left.{ }^{+} 16\right]$. There are many advantages of using a social network model for representing diffuse structures. It captures not only the overall spatial morphology and distribution of the protein pattern, but also intrinsically captures relationships between different spatial components of the pattern. Finally, by permitting the network to evolve over time, the changing properties of the social network can be interpreted biologically to describe different observed phenomena: just as "traditional" social networks evolve through the addition and deletion of connections between individuals, so do such events describe precisely how the morphology, both locally in one part of the cell, and globally across multiple cells, changes in response to stimuli.

We have begun by modeling the subcellular patterns of mitochondria in cervical epithelial (HeLa) cells. Mitochondria are dynamic organelles, which undergo continual rounds of fission 
and fusion. These fission and fusion events are important for maintaining proper function and overall mitochondrial health [ZLN13] [WL16]. Mitochondrial fission allows for the turnover of damaged and the protection of healthy organelles. Additionally, mitochondrial fusion leads to the mixing of internal contents, which is important for responding to environmental needs [ZLN13] [KPSBW08].

The dynamics between fission and fusion creates a spectrum of mitochondrial morphologies. Imbalances between fission and fusion events generate phenotypes associated with mitochondrial dysfunction [ZLN13]. An excess of fission or dearth of fusion events results in fragmented mitochondria; in this phenotype, the mitochondrial network is fractured, and individual mitochondria exist in small spheres. Conversely, an overabundance of fusion or a lack of fission events generate hyperfused mitochondria; in this phenotype, the mitochondrial network is overconnected, and composed of long interconnected tubules [CSCI $\left.{ }^{+} 08\right]$. Recently, several bacterial species have been shown to cause mitochondrial perturbations during infection $\left[\mathrm{SBS}^{+}{ }^{11}\right][\mathrm{FCGQR} 15]$. Such unique morphologies should be detectable at a quantitative level using social network modeling.

Through social network modeling, we hope to build a more rapid and efficient method for identifying changes in size, shape, and distribution of mitochondria as well as other diffuse organelles. In this work, we present a proof-of-concept pipeline which segments cells with fluorescent stains on the mitochondria for individual analysis. Once the cells are segmented, we use a Gaussian Mixture Model (GMM) to parameterize the spatial distribution of the mitochondrial protein patterns at evenly-spaced time intervals, and allow the GMM parameters to update smoothly from the previous time point to the next. Finally, we demonstrate how the learned parameters of the GMM can be used to construct social networks for representing the mitochondria. The complete pipeline can be seen in Fig. 1.

\section{Data}

We have constructed a library of live confocal imaging videos that display the full spectrum of mitochondrial morphologies in HeLa cells, from fragmented to hyperfused. To visualize the mitochondria, HeLa cells were stably transfected with DsRed2Mito-7 (DsRed2-HeLa), which fluorescently labels mitochondria with red emission spectra (a gift from Michael Davidson, Addgene plasmid \#55838). All of our videos were taken using a Nikon A1R Confocal. Cells were kept in an imaging chamber that maintained 37 degrees $\mathrm{C}$ and $5 \% \mathrm{CO} 2$ for the duration of imaging. The resonant scanning head was used to capture an image every ten seconds for the length of the video. The resulting time series videos have more than 20,000 frames per video. Each frame is of dimensions of 512x512 pixels (Fig. 2).

Wild type mitochondrial morphology was captured by imaging DsRed2-HeLa cells in typical growth medium (DMEM plus $10 \%$ fetal bovine serum) (Fig. 2, center). To generate the fragmented phenotype, cells were exposed to the pore-forming toxin listeriolysin O (LLO) at a final concentration of $6 \mathrm{nM}$ (Fig. 2, left). Mitochondrial hyperfusion was induced through the addition of mitochondria division inhibitor-1 (mdivi-1) at a final concentration of $50 \mu \mathrm{M}$ (Fig. 2, right). These subsets with different known qualitative phenotypes serve as bases upon which to condition our quantitative analyses.

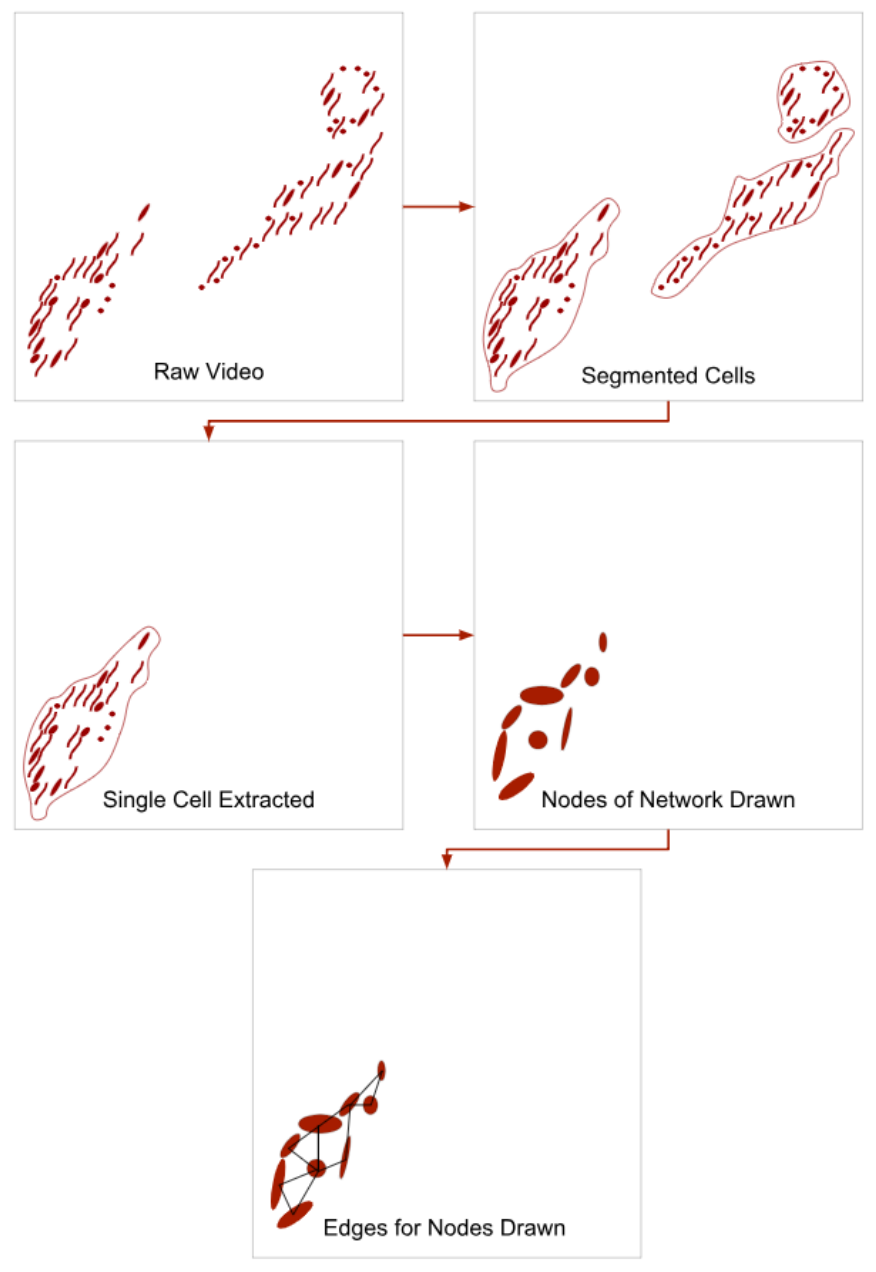

Fig. 1: An abstract representation of our proposed pipeline. The first frame represents the raw unsegmented image of mitochondria in three cells. The second frame demonstrates simultaneous segmentation, as a border is drawn around each cell. The third frame represents a single cell being extracted for analysis using the determined segmentation. The fourth frame shows a characteristic set of nodes determined by applying a mixture model to the distribution of fluorescent mitochondria. The final frame shows edges added to the nodes to complete the network structure. At this point in the pipeline, network analysis can be applied to the induced graph. These steps are applied to each frame of video allowing for fully temporal analysis. .

\section{Segmentation Pipeline}

In order to avoid systemic bias in our downstream analysis pipeline as a result of different videos containing a varied and unbounded number of cells, we chose to study each cell individually. This required segmenting each individual cell and studying its spatiotemporal dynamics in isolation from the others. While segmentation of cells from fluorescence or histology images is becoming very common, segmenting diffuse protein patterns-such as mitochondria--is much more challenging. We leveraged the fact that, given the small interval (10s) between frames of a video, overall movement between a given pair of subsequent frames would be minimal. We used deformable contours with slight updates from the previous frame to build out segmentation masks. However, the diffuse structure combined with the near overlap of cells in frames necessitated a "priming" of the segmentation pipeline with a hand-drawn mask at time 0 . We used 

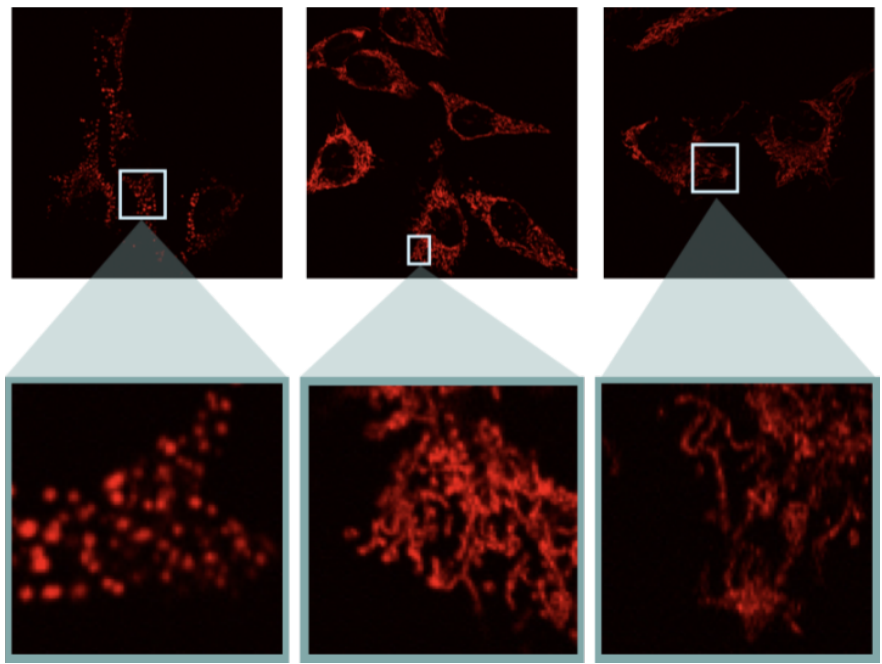

Fig. 2: Sample frames from each of the subsets of data. (Left) LLO induced mitochondrial fragmentation (Center) Wild type HeLa mitochondrial morphology (Right) Mdivi-1 induced mitochondrial hyperfusion

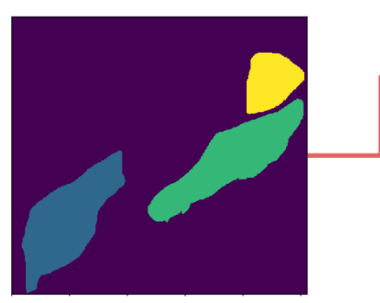

$\nabla$
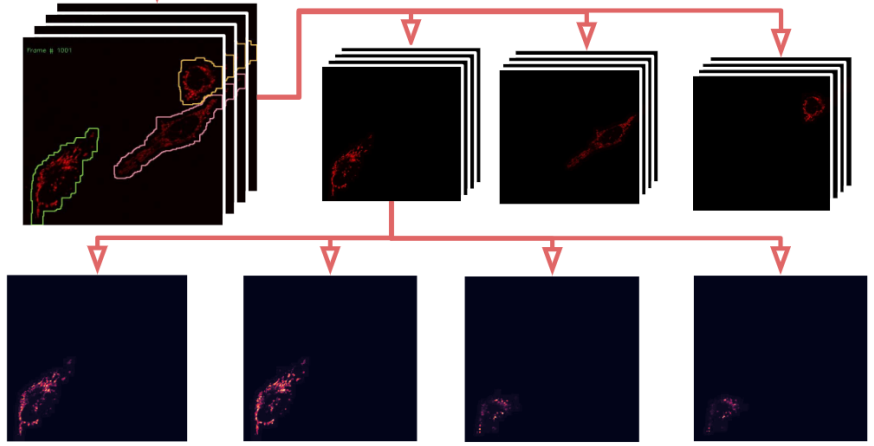

Fig. 3: Diagram of the cell segmentation process. (Top Left) Hand drawn masks of the first frame in VTK format were used to "seed" the deformable contours. (Top Right) A series of frames from a single video with autonomously drawn contours. (Middle) Stack of frames from a single video converted to separate videos for each cell. (Bottom) single cell video unraveled as grayscale image for frame by frame network modeling.

the ITK-SNAP software [YPCH $\left.{ }^{+} 06\right]$ to label each cell manually in the first frame of each video, generating a VTK file with the segmentation maps (Fig. 3, top left).

Our segmentation process used these maps as "seeds", updating the maps at each frame of the video using deformable contours: iterative dilation, thresholding, and contour detection process over the entire video, resulting in a set of masks for each frame and each cell in the frame. These masks could then be used to pull out individual cells over the course of the video (Fig. 3).

While this process was very effective at following the cells, occasionally the model would lose small areas of mitochondrial
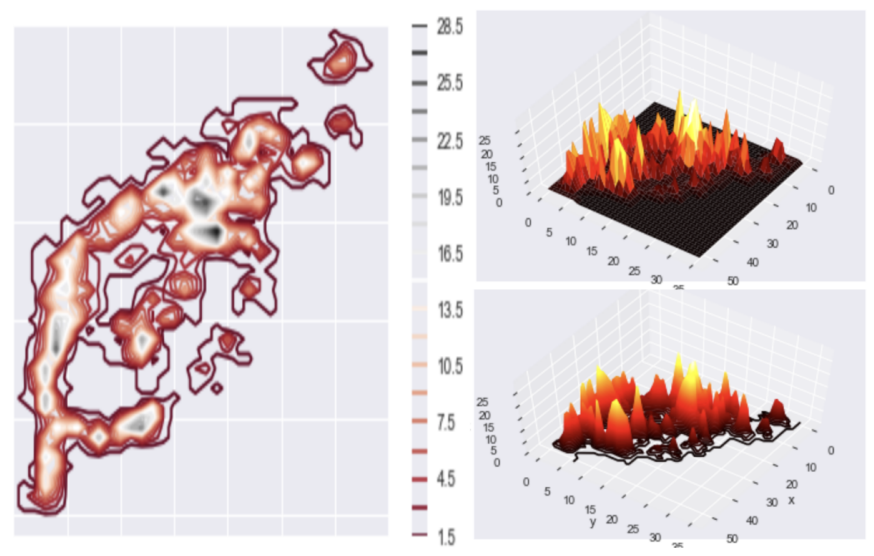

Fig. 4: (Left) a 2D probability representation of the intensity of a sample cell. (Top Right) the Intensity map of the image in a $3 D$ representation. (Bottom Right) the $3 D$ contour of the same cell.

mass which was sufficiently far away from the more contiguous structure. To compensate, we added a final process of iterative dilation to prevent loss and give a more generous contour. With these adjustments, we ran into a rare problem of cell contact or overlap. In response, we continued the iterative dilation with more iterations and smaller dilations checking for overlap with another map each iteration. In the case of an overlap, which would only be a few pixels with the small dilation kernel, we used a simple XOR to remove the few overlapping pixels while still allowing the mask to expand in areas unclaimed by other cells. With this case being rare, we found the process mostly followed any visible boundary of the adjacent cell.

The output of this step was the individual cell masks, one for each cell at each frame, providing a complete segmentation of each cell.

\section{Social Network Engineering}

To induce a network structure over the mitochondrial patterns of the segmented cells, we used a Gaussian Mixture Model (GMM). The means and covariances of the model components would represent two critical features of a social network: the individual nodes (means), and the nodes' relationships to each other (covariances). An independent model would be trained for each individual cell, and the model parameters would be permitted to evolve over the course of the videos to capture the changing underlying morphologies.

We first applied a Gaussian smoothing filter to minimize or eliminate artifacts in the video images. We then converted the frames of the video to a discrete probability distribution by normalizing the grayscale pixel intensities to sum to 1 (Fig. 4). Following the conversion to a probability density, we counted local pixel maxima and used these points--both the number of maxima found, and their spatial locations--as the initial components our GMM. These components were fed into the GMM fit() procedure in scikit-learn (Fig. 5). The learned GMM components would minimize the disparity between the joint probability density of the GMM, and the original empirical probability density of the image, parameterizing the structure of the mitochondrial pattern. Using the learned components as nodes in the final network allow for the network structure to be learned purely from the mitochondrial topology. 
The code for converting a single image frame to a discrete probability density function and learn the initial GMM components are as follows:

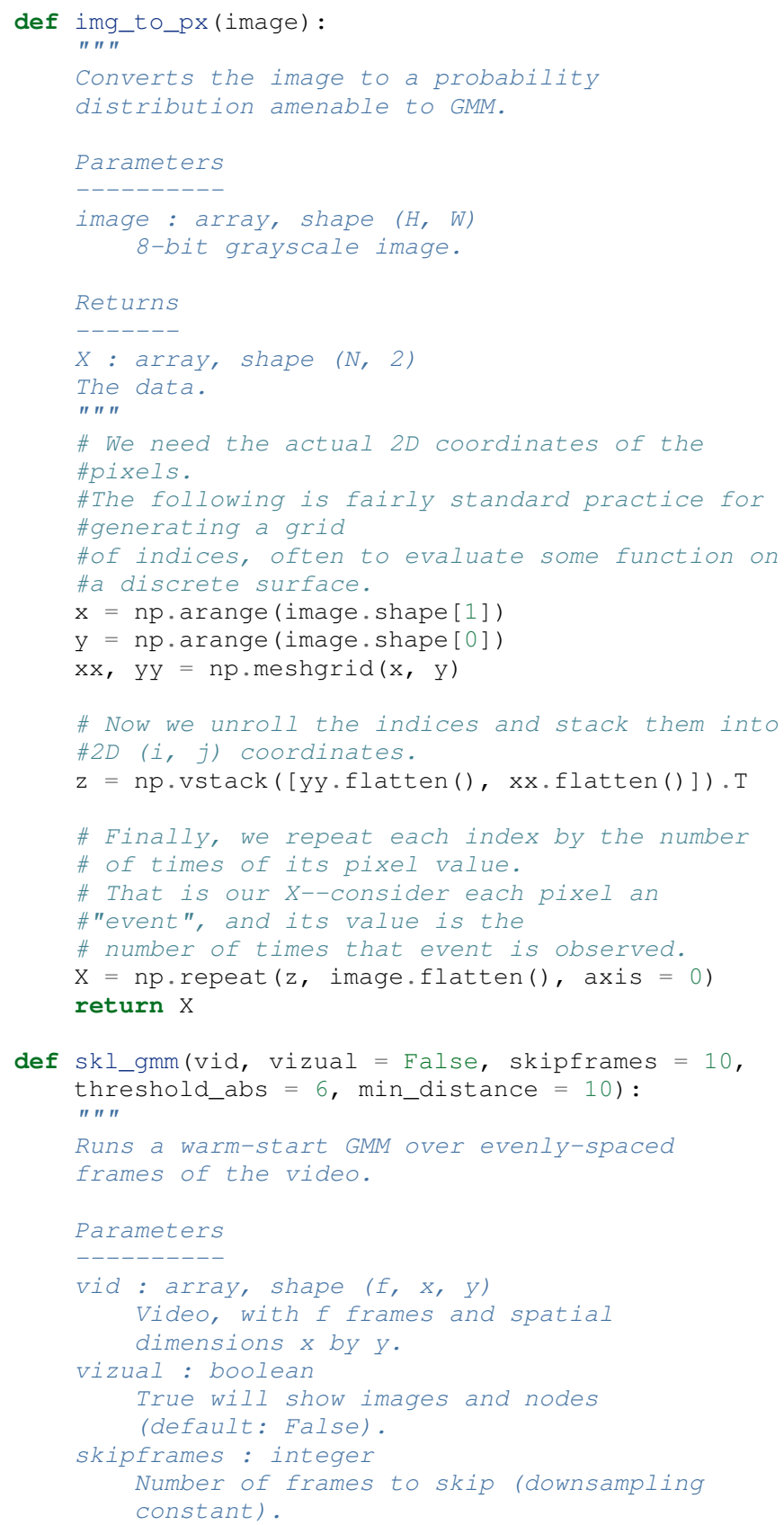

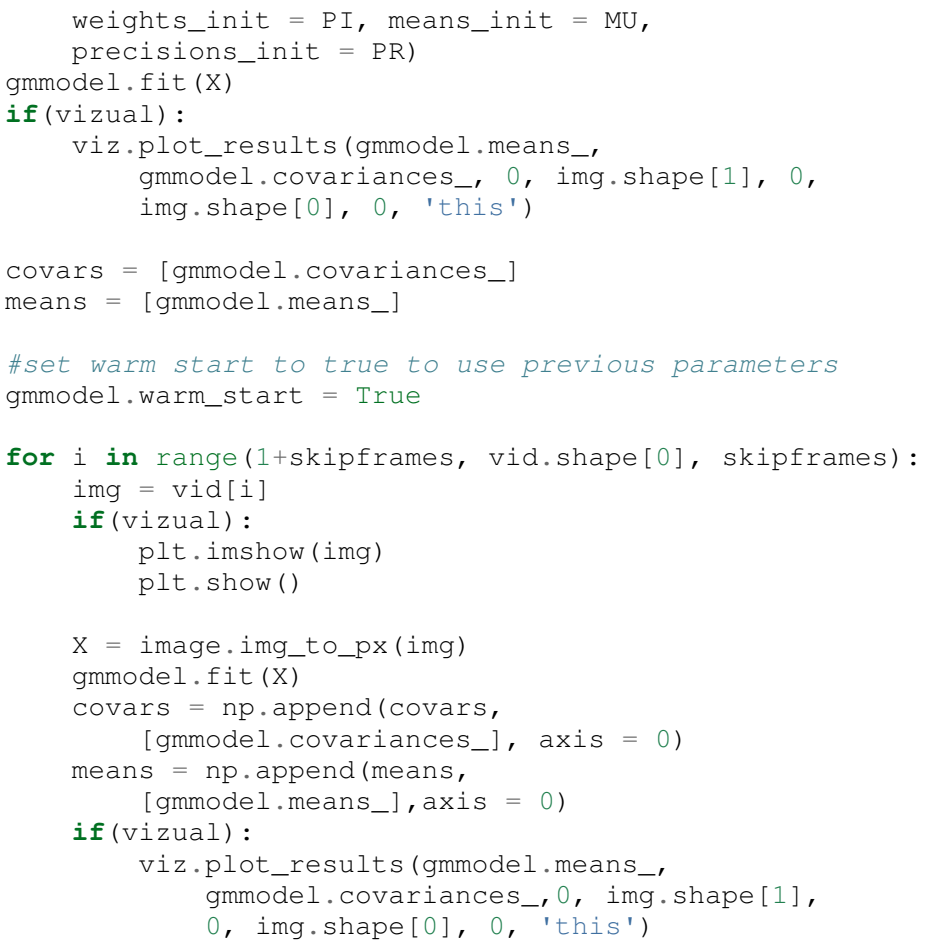

return means, covars
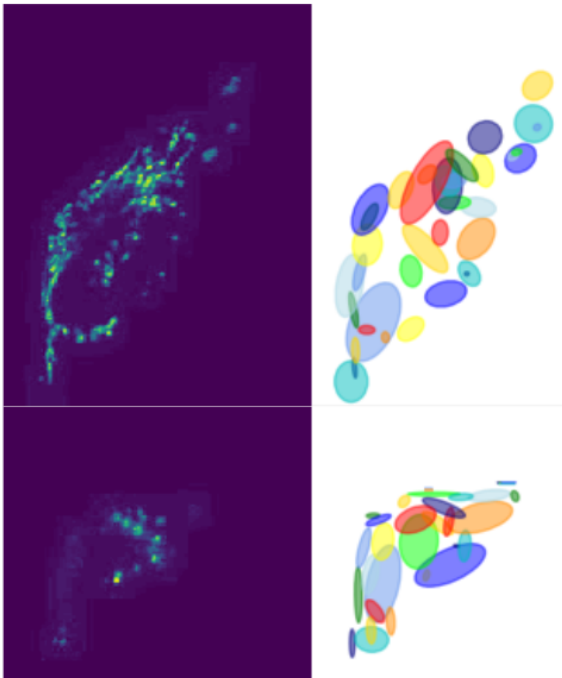

Fig. 5: A cell (Left) and the nodes (Right) as generated by a gaussian mixture model for the first (Rop) and last (Rottom) frames of a video showing a cell fragmented by LLO

For connecting the nodes with weighted edges, we explored multiple approaches that balanced realistically encapsulating the underlying biology (i.e., did not create connections between uncorrelated objects) and computational tractability. Initially, we chose a manual distance threshold and used this as the "neighborhood size" for the radial-basis function, a common connectionweighting metric that varies smoothly from 0 (not connected) to 1 (fully connected), and is a function of the Euclidean distance between the two nodes, weighted by the neighborhood size. A second attempt to make this process more data-driven was to replace the manually-crafted neighborhood size with the Gaussian covariance in the direction of the node to be connected $(6$, mid). In both cases, to avoid fully-connected graphs and induce some 


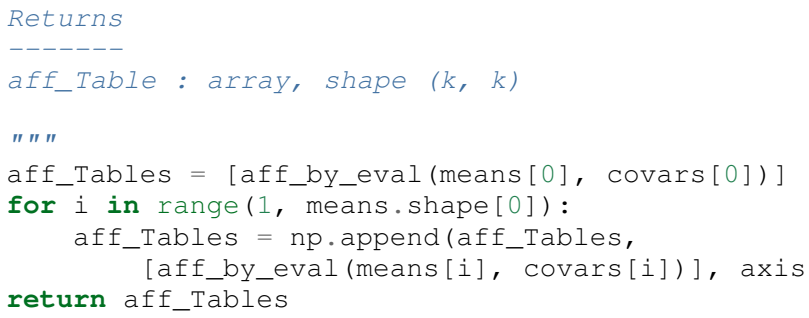

\section{Current Insights and Future Work Discussion}

After building networks using the described GMM method for each cell under varying conditions (control/wildtype, LLO, mdivi), we have qualitatively observed systemic differences in the learned model parameters that would separate these conditions. Interestingly, as the mitochondria fragment (i.e., LLO), the GMM components become more strongly connected, not less (7). We attribute this to a misinformed intuition: as the mitochondria fragment and the underlying probability density function becomes more uniform, the GMM components will likewise become more uniform, resulting in a more uniformly connected network. The overall number of connections also increases, as the cells tend to collapse at the same time as mitochondrial fragmentation, resulting in the same number of GMM components spatially colocating in a much smaller space, effectively "forcing" connections by virtue of proximity. By comparison, the control cell shows much less variation in the distribution of network connectivity and edge weights over time; this reflects a relatively stable social network, unperturbed by external stimuli.

The next step, then, is to develop a temporal model of the GMM component evolution in terms of the social network. This would take the form of a series of graph Laplacians and observing how the Laplacians change, likely as a function of Laplacian gradients. This would highlight specific portions of the social networks that covary over space and time; in other words, it would provide insight into the coordinated fragmentation or hyperfusion of the mitochondria in response to the provided stimulus. These features could then be incorporated into a broader supervised learning pipeline to distinguish patterns and discern the effects of an unknown stimulant (e.g., drug discovery), or an unsupervised learning pipeline to identify all observed mitochondrial phenotypes.

Additional methods of analyzing the graph structure of the social network would help to determine specific phenotypic changes induced by certain stimuli. In particular, classic graph metrics such as connectivity, cliques, and eigenvector centrality would help to precisely measure the global effects of certain stimuli on the mitochondria. Other algorithms, such as spectral clustering or PageRank for global network analysis from local phenomena would provide intuition into the local changes in mitochondrial phenotype responsible for inducing the global structure. These features would be invaluable for characterizing certain specific cell-wide or even organism-wide conditions.

We also aim to improve the process through which the social network is constructed in the first place. The incorporation of a single uniform component into the overall GMM would provide a robust method of accounting for background noise in the form of a learned, data-driven threshold. Additional refinements of the affinity function that determines the existence of connections between nodes, and their weight and direction, will be pursued: the Kullback-Leibler (KL) divergence is a popular method for measuring the difference between two probability distributions, and would be a natural fit for evaluating how similar two GMM components are.

In this paper, we have presented a proof-of-concept for parameterizing and modeling spatiotemporal changes in diffuse subcellular protein patterns using GMMs. We have presented how the learned parameters of the GMM can be updated to account for changing biological phenotypes, and how these parameters can then be used to induce a social network of interacting nodes. Finally, we show how the properties of the social network can be interpreted to provide biological insights, in particular how the underlying system may be responding to some kind of stimulus. This has potential implications in fundamental biology and translational biomedicine; we aim to complete our analysis package and release it as open source for the research community to use in the near future.

\section{Acknowledgments}

This project was supported in part by a grant from the National Science Foundation (\#1458766).

\section{REFERENCES}

$\left[\mathrm{CSCI}^{+}\right.$08] Ann Cassidy-Stone, Jerry E Chipuk, Elena Ingerman, Cheng Song, Choong Yoo, Tomomi Kuwana, Mark J Kurth, Jared T Shaw, Jenny E Hinshaw, Douglas R Green, et al. Chemical inhibition of the mitochondrial division dynamin reveals its role in bax/bak-dependent mitochondrial outer membrane permeabilization. Developmental cell, 14(2):193-204, 2008.

$\left[\mathrm{DGD}^{+}\right.$16] Andrew T Drysdale, Logan Grosenick, Jonathan Downar, Katharine Dunlop, Farrokh Mansouri, Yue Meng, Robert N Fetcho, Benjamin Zebley, Desmond J Oathes, Amit Etkin, Alan F Schatzberg, Keith Sudheimer, Jennifer Keller, Helen S Mayberg, Faith M Gunning, George S Alexopoulos, Michael D Fox, Alvaro Pascual-Leone, Henning U Voss, BJ Casey, Marc J Dubin, and Conor Liston. Resting-state connectivity biomarkers define neurophysiological subtypes of depression. $\mathrm{Na}$ ture Medicine, 2016. URL: http://dx.doi.org/10.1038/nm.4246, doi:10.1038/nm.4246.

[FCGQR15] Kari Fine-Coulson, Steeve Giguère, Frederick D Quinn, and Barbara J Reaves. Infection of a549 human type ii epithelial cells with mycobacterium tuberculosis induces changes in mitochondrial morphology, distribution and mass that are dependent on the early secreted antigen, esat-6. Microbes and infection, 17(10):689-697, 2015.

[KPSBW08] Andrew B Knott, Guy Perkins, Robert Schwarzenbacher, and Ella Bossy-Wetzel. Mitochondrial fragmentation in neurodegeneration. Nature Reviews Neuroscience, 9(7):505, 2008.

[SBS $\left.{ }^{+} 11\right] \quad$ Fabrizia Stavru, Frédéric Bouillaud, Anna Sartori, Daniel Ricquier, and Pascale Cossart. Listeria monocytogenes transiently alters mitochondrial dynamics during infection. Proceedings of the National Academy of Sciences, 108(9):3612-3617, 2011.

[STS17] Valentine Svensson, Sarah A. Teichmann, and Oliver Stegle. Spatialde - identification of spatially variable genes. bioRxiv, 2017. URL: https://www.biorxiv.org/content/early/2017/ 11/08/143321, arXiv:https://www.biorxiv.org/ content/early/2017/11/08/143321.full.pdf, doi:10.1101/143321.

[VCL $^{+}$17] Alex M Valm, Sarah Cohen, Wesley R Legant, Justin Melunis, Uri Hershberg, Eric Wait, Andrew R Cohen, Michael W Davidson, Eric Betzig, and Jennifer Lippincott-Schwartz. Applying systems-level spectral imaging and analysis to reveal the organelle interactome. Nature, 546(7656):162, 2017.

[WL16] Timothy Wai and Thomas Langer. Mitochondrial dynamics and metabolic regulation. Trends in Endocrinology \& Metabolism, 27(2):105-117, 2016

[YPCH ${ }^{+}$06] Paul A. Yushkevich, Joseph Piven, Heather Cody Hazlett, Rachel Gimpel Smith, Sean Ho, James C. Gee, and Guido Gerig. Userguided 3D active contour segmentation of anatomical structures: Significantly improved efficiency and reliability. Neuroimage, 31(3):1116-1128, 2006. 
Frame 0
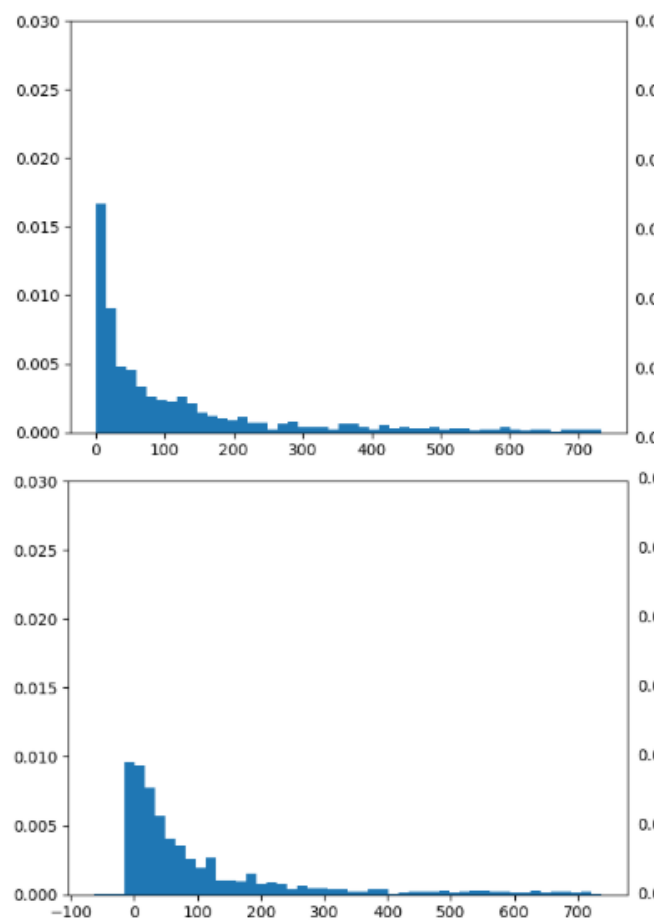

Frame 60
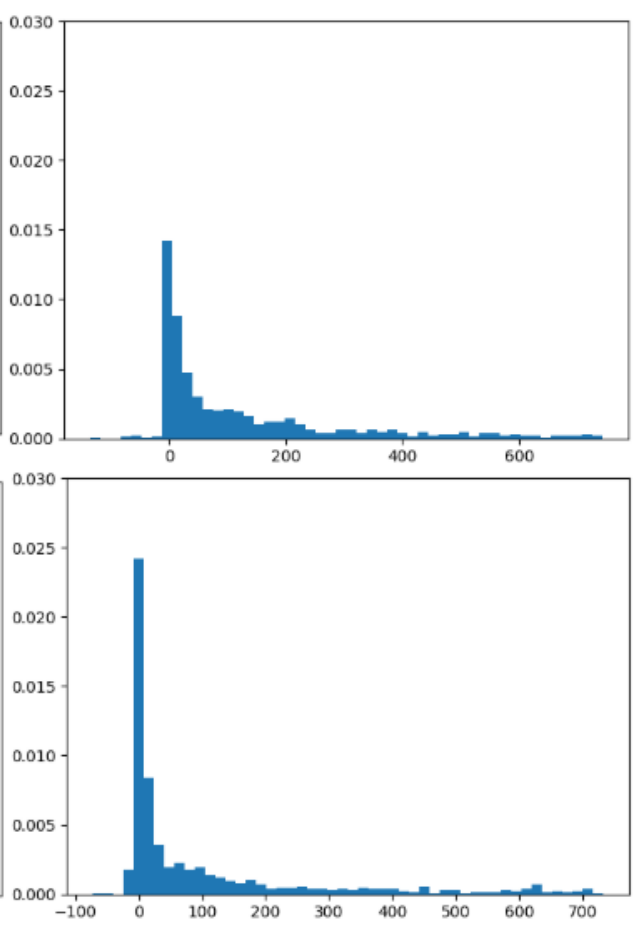

Frame 130
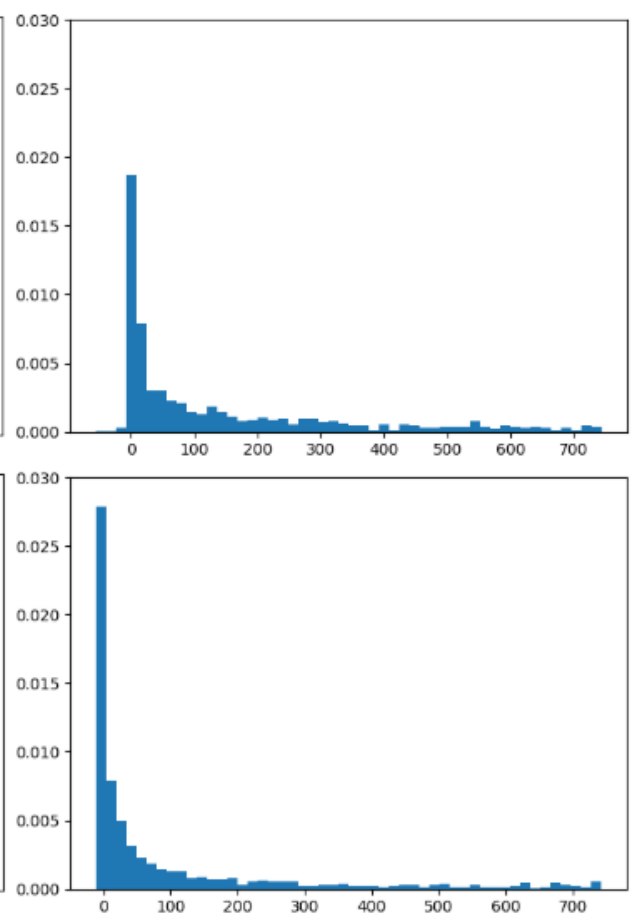

Fig. 7: A series of distribution plots of the negative log of values found in six affinity tables developed using the model learned at an early, middle, and late video frame. (Top) The tables generated from a control cell which show little variation in distribution. (Bottom) The tables generated from the LLO cell which shows a drastic increase in connectivity over time as the cell fragments.

[ZLN13] Jian Zhao, Urban Lendahl, and Monica Nistér. Regulation of mitochondrial dynamics: convergences and divergences between yeast and vertebrates. Cellular and molecular life sciences, 70(6):951-976, 2013. 